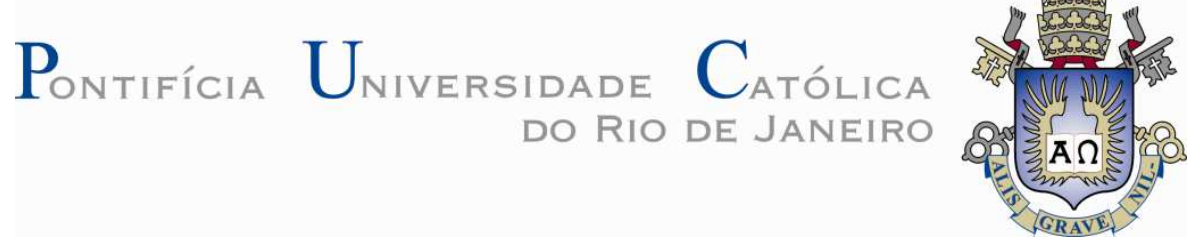

José Miguel Mayta Tito

\title{
Simulação de Trocadores de Calor de Placas para Sistemas de Refrigeração em Cascata
}

Orientador: Professor José Alberto dos Reis Parise Co-orientador: Dr. Samuel Fortunato Yana Motta 


\section{Simulação de Trocadores de Calor de Placas para Sistemas de Refrigeração em Cascata}

Dissertação apresentada como requisito parcial para obtenção do título de Mestre pelo Programa de PósGraduação em Engenharia Mecânica da PUC-Rio. Aprovada pela Comissão Examinadora abaixo assinada.

Prof. José Alberto dos Reis Parise

Orientador

Pontifícia Universidade Católica do Rio de Janeiro

Dr. Samuel Fortunato Yana Motta

Co-orientador

Prof. Carlos Valois Braga

Pontifícia Universidade Católica do Rio de Janeiro

Prof. Sergio Leal Braga

Pontifícia Universidade Católica do Rio de Janeiro

Prof. Gherhardt Ribatski

Universidade de São Paulo

Prof. José Eugenio Leal Coordenador Setorial do Centro

Técnico Científico - PUC-Rio

Rio de Janeiro, 31 de Maio de 2011 
Todos os direitos reservados. É proibida a reprodução total ou parcial do trabalho sem autorização da universidade, do autor e do orientador.

\section{José Miguel Mayta Tito}

Graduou-se em Engenharia Mecânica no Dpto. de Engenharia Mecânica da Universidad Nacional de San Agustin, Arequipa - Perú no ano de 2008. Atualmente trabalhando na simulação de equipamento de refrigeração.

Ficha Catalográfica

Tito, José Miguel Mayta

Simulação de trocadores de calor de placas para sistemas de refrigeração em cascata / José Miguel Mayta Tito ; orientador: José Alberto dos Reis Parise ; co-orientador: Samuel Fortunato Yana Motta. $-2011$.

151 f. : il. (color.) ; $30 \mathrm{~cm}$

Dissertação (mestrado)-Pontifícia Universidade Católica do Rio de Janeiro, Departamento de Engenharia Mecânica, 2011.

Inclui bibliografia

1. Engenharia mecânica - Teses. 2. Refrigeração. 3. Simulação. 4. Trocadores de calor de placas soldadas. 5. Sistema em cascata. 6. Refrigerantes. I. Parise, José Alberto dos Reis. II. Motta, Samuel Fortunato Yana. III. Pontifícia Universidade Católica do Rio de Janeiro. Departamento de Engenharia Mecânica. IV. Título. 
"Paciência e perseverança têm o efeito mágico de fazer as dificuldades desaparecerem e os obstáculos sumirem" 


\section{Agradecimentos}

A Deus, pela graça de ter me permitido concluir este trabalho.

À minha família, meus pais Miguel e Juana e meus irmãos Rosa, Magaly, Pilar e Julio pelo amor, o apoio incondicional e ânimos no transcurso destes dois anos.

A José Alberto dos Reis Parise, meu orientador, pela amizade e enorme paciência que teve comigo durante estes dois anos.

A Samuel Fortunato Yan Motta, meu co-orientador pela motivação e pela confiança que teve comigo no desenvolvimento desta dissertação para levá-la a cabo.

A Paul Ortega Sotomayor, pela amizade e apoio na minha dissertação desde o começo.

A meus amigos Marco, Carlos, Andres, Luis F., Darwin, Andrea, Lorena Leydi e Sandra pela amizade e pelos bons momentos compartilhados nestes anos.

Agradecimentos em particular para os órgãos de fomento à pesquisa CNPq, CAPES e FAPERJ, pelo apoio financeiro fornecido, sem o qual este trabalho não teria ser possível. Por outro lado, meu agradecimento é também dirigido à empresa Honeywell Inc. pelo apoio financeiro ao projeto. 


\section{Resumo}

Mayta Tito, José Miguel; dos Reis Parise, José Alberto. Simulação de trocadores de calor de placas para sistemas de refrigeração em cascata. Rio de Janeiro, 2011. 151p. Dissertação de Mestrado - Departamento de Engenharia Mecânica, Pontifícia Universidade Católica do Rio de Janeiro.

Desenvolveu-se um modelo de simulação para trocadores de calor de placas soldadas (BPHE - "Brazed Plate Heat Exchanger") operando em regime permanente em um sistema de refrigeração em cascata por compressão de vapor, ou seja, condensador, trocador de calor intermediário (ou "condensador em cascata") e evaporador. O modelo adota o método de análise local, onde o trocador de calor é dividido em uma série de pequenos volumes de controle, para os quais as equações de troca de calor e de conservação de massa e de energia são aplicadas. Para o calculo dos coeficientes locais de transferência de calor e fator de atrito foram utilizadas correlações disponíveis na literatura, considerando as regiões de escoamento monofásico ou bifásico em cada um dos trocadores, Estas correlações cobrem valores de fluxo de calor entre $2,5 \mathrm{~kW} / \mathrm{m}^{2}$ e $185 \mathrm{~kW} / \mathrm{m}^{2}$, temperaturas de saturação entre $5^{\circ} \mathrm{C}$ e $30^{\circ} \mathrm{C}$, e aplicam-se a geometrias com ângulos de corrugação entre 20 e 60 graus. Um programa computacional foi desenvolvido em FORTRAN para o cálculo do desempenho térmico dos trocadores de calor e das correlações de as[ida dos dois fluidos. Na simulação foram consideradas conhecidas as condições de entrada dos fluidos e a geometria do trocador. As propriedades termo-físicas dos fluidos foram calculadas utilizandose a mais recente versão do padrão NIST de referência de propriedades termodinâmicas e de transporte (REFPROP 9.0), permitindo a simulação dos trocadores de calor operando com uma vasta gama de refrigerantes. Os resultados da simulação foram comparados com os dados experimentais (condensador e evaporador) levantados por outros autores para os refrigerantes R22 e R290, tendose obtido boa concordância. Uma analise de sensibilidade para os trocadores de calor, utilizando os novos refrigerantes R1234yf e R1234ze, foi também realizada.

\section{Palavras-chave}

Refrigeração, simulação, trocadores de calor de placas soldadas, sistema em cascata, refrigerantes. 


\section{Abstract}

Mayta Tito, José Miguel;, dos Reis Parise, José Alberto. Simulation of brazed plate heat exchangers for cascade vapor compression sistem, Rio de Janeiro, 2011. 151p. MSc. Dissertation - Departamento de Engenharia Mecânica, Pontifícia Universidade Católica do Rio de Janeiro.

A simulation model of brazed plate heat exchangers (BPHE) operating in steady-state in a cascade vapor compression refrigeration system has been developed. For this system the heat exchangers were the condenser, intermediate heat exchanger or cascade-condenser and evaporator. The model adopts a local analysis method, where the heat exchanger is divided into a series of small control volumes, to which the heat transfer rate equations and the fundamental of conservation of mass and energy equation. Local heat exchanger coefficients and friction factor are calculated using correlations available in literature, considering regions of single-phase or twophase flow for each one of the heat exchangers. These correlations have heat flux values ranging from $2,5 \mathrm{~kW} / \mathrm{m}^{2}$ to $185 \mathrm{~kW} / \mathrm{m}^{2}$, saturation temperatures from $5^{\circ} \mathrm{C}$ to $35^{\circ} \mathrm{C}$ and were applied to geometries with corrugation angle ranging from $20^{\circ}$ to $60^{\circ}$.In order to calculate the thermal performance of the heat exchangers and the output conditions of the two fluids a computational program was developed in FORTRAN. This simulation considers known inlet conditions of the fluids and the geometry of the heat exchanger. The thermophysical properties of the refrigerants fluids were calculated using the version 7 of REFPROP, a package by NIST (National Institute of Standards and Technology), that allow for the simulation of heat exchangers with a wide operating range of refrigerants. The simulation results were compared with experimental data (condenser and evaporator) for R22 and R290 refrigerants, obtaining a good agreement. A sensibility analysis for heat exchangers, using the new R1234yf and R1234ze has also been carried out.

\section{Keywords}

Refrigeration, Simulation, brazed plate heat exchangers, cascade system, refrigerants 


\section{Sumário}

1 Introdução 22

1.1. A questão ambiental 22

1.2. Trocadores de calor de placas 24

1.3. Sistemas de refrigeração em cascata 26

$\begin{array}{ll}\text { 1.4. Justificativa } & 27\end{array}$

1.5. Objetivo do trabalho 28

1.6. Conteúdo do trabalho 29

2 Revisão Bibliográfica $\quad 31$

2.1. Introdução 31

2.2. Simulações numéricas - Troca de calor monofásica 31

2.3. Simulações numéricas - Troca de calor com mudança de fase 36

2.4. Estudos experimentais 38

2.5. Sistema de refrigeração em cascata 42

2.6. Conclusões da revisão bibliográfica. 45

3 Modelo Matemático 48

3.1. Análise do volume de controle 48

3.2. Geometria da placa 49

3.3. Equações de conservação $\quad 51$

3.4. Hipóteses simplificadoras para trocadores de calor a placas 52

3.5. Equações de transferência de calor 53

3.6. Queda de pressão nos trocadores de calor a placas 56

3.7. Números adimensionais $\quad 58$

3.8. Condensador 60

3.8.1. Coeficiente local de transferência de calor para a condensação 61

3.8.2. Fator de atrito para a condensação 64

3.9. Evaporador 64

3.9.1. Coeficiente local de transferência de calor para a ebulição 65

3.9.2. Fator de atrito para a evaporação 69 
3.10. Condensador de Cascata ou Trocador intermediário

3.11. Coeficiente de troca de calor para as regiões monofásicas $\quad 70$

3.12. Fator de atrito para o escoamento monofásico 72

4 Método de Solução 73

4.1. Procedimento de solução 73

4.2. Algoritmo de solução para o condensador 76

4.3. Algoritmo de solução para o evaporador 79

4.4. Algoritmo de solução para o condensador cascata 81

4.5. Critério de convergência 84

4.6. Método de relaxação 85

4.7. Programa computacional 86

5 Resultados 74

5.1. Teste de malha 74

5.2. Validação dos modelos 90

5.3. Dados experimentais $\quad 91$

5.3.1. Dados experimentais para o R22 e R290 no condensador $\quad 91$

5.3.2. Dados experimentais para o R22 e R290 no evaporador 95

5.4. Comparação com dados experimentais 99

5.4.1. Simulação numérica do condensador 101

5.4.1.1. Resultados da simulação do condensador com o refrigerante R22 102

5.4.1.2. Resultados da simulação do condensador com o refrigerante R290 106

5.4.2. Simulação numérica do evaporador $\quad 110$

5.4.2.1. Resultados da simulação do evaporador com o refrigerante R22 110

5.4.2.2. Resultados da simulação do evaporador com o refrigerante R290 114

5.5. Comparação com dados do fabricante 118

5.5.1. Simulação numérica do condensador cascata 120

5.5.2. Comparação com o software SSP G7 121

5.5.3. Resultados da simulação do condensador em cascata 121 
6 Conclusões e sugestões 129

6.1. Conclusões 129

6.2. Sugestões para trabalhos futuros 130

Referências Bibliográficas 131

APÊNDICE A

A.1 Resultados da simulação do condensador com o refrigerante $\mathrm{R} 22$

A.2 Resultados da simulação do condensador com o refrigerante R290

A.3 Resultados da simulação do evaporador com o refrigerante R22

A.4 Resultados da simulação do evaporador com o refrigerante R290 


\section{Lista de figuras}

Figura 1 Breve história e evolução dos refrigerantes ao longo dos anos (Calm,2008).

Figura 2 Típico trocador de calor de placas brazadas

(Wang et al. 2007).

Figura 3 Parâmetros físicos e princípio de funcionamento de um BPHE (Stenhede,2001).

Figura 4 Esquema do sistema de refrigeração em cascata.

(Bayrakci, 2010).

Figura 5 Diagrama $\mathrm{P}$-h para um sistema de refrigeração em cascata teórico. (Lee et al. 2006).

Figura 6 Trocador de calor de placas e volume de controle elementar. 48 Figura 7 (a) Condições de entrada e de saída do refrigerante e do fluido de transferência de calor no volume de controle elementar.

(b) Esquema real e dimensões do volume de controle elementar.

Figura 8 (a) Parâmetros geométricos do tipo placa Chevron

(b) Seção transversal e geometria do canal (Kakaç e Liu, 2002).

Figura 9 Circuito térmico equivalente para a troca de calor em determinado trecho de um PHE. 55

Figura 10. Diagrama de temperaturas no condensador.

Figura 11 - (a) Condensador de placas - Marca Alfa Laval.

(b) Representação esquemática do condensador de placas.

Figura 12. Diagrama de temperaturas para os PHEs usados como evaporador.

Figura 13. (a) Evaporador de placas - Alfa Laval. (b) Representação esquemática dos fluxos no evaporador de placas.

Figura 14. Diagrama de temperaturas para os PHEs usados como condensador cascata.

Figura 15 Diagrama de percorrido dos fluidos no condensador.

Figura 16 Diagrama de percorrido dos fluidos no evaporador.

Figura 17 Diagrama de temperaturas dos fluidos no 
trocador intermediário e sentido de cálculo do trocador de calor.

Figura 18 Teste de malha para o condensador de placas. 88

Figura 19 Teste de malha para o evaporador de placas. $\quad 89$

Figura 20 Teste de malha para o trocador intermediário de placas. $\quad 90$

Figura 21 Comparação de dados da taxa total de transferência de calor numérica com a experimental.

Figura 22 Erro relativo da taxa total de transferência de calor versus a vazão mássica do refrigerante no condensador.

Figura 23 Erro da temperatura de saída do refrigerante versus a vazão mássica do refrigerante no condensador.

Figura 24 Erro da temperatura de saída do fluido de resfriamento versus a vazão mássica do fluido de resfriamento no condensador.

Figura 25 Erro de UA versus a vazão mássica do refrigerante no condensador.

Figura 26 Comparação de dados da taxa de transferência de calor numérica com a experimental.

Figura 27 Erro relativo da taxa de transferência de calor versus a vazão mássica do refrigerante no condensador.

Figura 28 Erro da temperatura de saída do refrigerante versus a vazão mássica do refrigerante no condensador.

Figura 29 Erro da temperatura de saída do fluido de resfriamento versus a vazão mássica para o condensador.

Figura 30 Erro de UA versus a vazão mássica do refrigerante no condensador.

Figura 31 Comparação de dados da taxa de transferência de calor numéricos com os experimentais.

Figura 32 Erro relativo da taxa de transferência de calor versus a vazão mássica do refrigerante para o evaporador.

Figura 33 Erro da temperatura de saída do refrigerante versus a vazão mássica do refrigerante no evaporador.

Figura 34 Erro da temperatura de saída do fluido secundário versus a vazão mássica para o evaporador.

Figura 35 Erro de UA versus a vazão mássica do refrigerante 
para o evaporador.

Figura 36 Comparação entre dados numéricos e experimentais da taxa de transferência de calor.

Figura 37 Erro relativo da taxa de transferência de calor versus a vazão mássica do refrigerante no evaporador.

Figura 38 Erro da temperatura de saída do refrigerante versus vazão mássica do refrigerante no evaporador.

Figura 39 Erro da temperatura de saída do fluido secundário versus a vazão mássica do fluido secundário no evaporador.

Figura 40 Erro de UA versus a vazão mássica do refrigerante no evaporador.

Figura 41 Perfis das temperaturas dos refrigerantes em função da posição do elemento no trocador de calor intermediário.

Figura 42 Variação dos coeficientes de transferência ao longo do trocador de calor intermediário.

Figura 43 Variação do titulo de vapor ao longo do trocador de calor intermediário.

Figura 44 Taxa de transferência de calor versus vazão mássica do fluido secundário.

Figura 45 Temperatura de saída do refrigerante versus a vazão mássica do fluido secundário.

Figura 46 Condutância versus a vazão mássica do fluido secundário.127

Figura 47 Queda de pressão versus vazão mássica do fluido secundário.

Figura 48 Coeficiente de transferência de calor local versus título de vapor.

Figura 49 Comparação das temperaturas de saída numérica e experimental do refrigerante R22 no condensador.

Figura 50 Comparação das temperaturas de saída numérica e experimental do fluido de resfriamento no condensador.

Figura 51 Comparação da condutância numérica e experimental.

Figura 52 Comparação das temperaturas de saída numérica e experimental do refrigerante R290 no condensador. 
Figura 53 Comparação das temperaturas de saída numérica e experimental do fluido de resfriamento no condensador.

Figura 54 Comparação das condutâncias numérica e experimental no condensador.

Figura 55 Comparação das temperaturas de saída numérica e experimental do refrigerante no condensador.

Figura 56 Comparação das temperaturas de saída numérica e experimental do fluido secundário no evaporador.

Figura 57 Comparação das condutâncias no evaporador.

Figura 58 Comparação das temperaturas de saída numérica e experimental do refrigerante.

Figura 59 Comparação das temperaturas de saída numérica e experimental do fluido secundário.

Figura 60 Comparação das condutâncias numérica e experimental no evaporador. 


\section{Lista de tabelas}

Tabela 1 Correlações empíricas para o coeficiente de transferência de calor em condensação e suas condições de operação.

Tabela 2 Correlações empíricas para o coeficiente de transferência de calor e suas condições de operação na evaporação.

Tabela 3- Correlações para o coeficiente de transferência de calor para escoamento monofásico em trocadores de calor de placas.

Tabela 4 Dados gerais do trocador de calor de placas soldadas utilizado para o condensador e evaporador (NIST, 1999).

Tabela 5 Condições de operação do refrigerante R22 no condensador (NIST, 1999).

Tabela 6 Condições de operação do fluido de resfriamento no condensador (NIST, 1999).

Tabela 7 Condições de operação do refrigerante R290 no condensador (NIST, 1999).

Tabela 8 Condições de operação do fluido de resfriamento no condensador (NIST, 1999).

Tabela 9 Condições de operação do refrigerante R22 no evaporador (NIST, 1999).

Tabela 10 Condições de operação do fluido secundário no evaporador (NIST, 1999).

Tabela 11 Condições de operação do refrigerante R290 no evaporador (NIST, 1999).

Tabela 12 Condições de operação do fluido secundário no evaporador (NIST, 1999).

Tabela 13 Combinações das correlações de transferência de calor e queda de pressão na região bifásica para o condensador.

Tabela 14 Combinações das correlações de transferência de calor e queda de pressão para o evaporador.

Tabela 15 Dados de entrada no condensador de placas.

Tabela 16 Erro médio das combinações na simulação 
do condensador de placas para o refrigerante R22.

Tabela 17 Erro médio das combinações das correlações

na simulação do condensador para o refrigerante R290.

Tabela 18 Dados de entrada do evaporador de placas.

Tabela 19 Erro médio das combinações das correlações

na simulação do evaporador de placas para o refrigerante R22.

Tabela 20 Erro médio das correlações na simulação do evaporador de placas para o refrigerante R290.

Tabela 21 Condições de operação para o software SSP G7.

Tabela 22 Parâmetros obtidos do software SSP G7.

Tabela 23 Dados gerais do trocador de calor de placas utilizado como condensador em cascata.

Tabela 24 Dados de entrada do condensador em cascata de placas. 120

Tabela 25 Condições de entrada para os refrigerantes.

Tabela 26 Comparação de dados obtidos das simulações.

Tabela 27 Estados termodinâmicos para o R22 no evaporador.

Tabela 28 Dados gerais do trocador de calor de placas soladas para o evaporador.

Tabela 29 Condições iniciais de operação do evaporador. 


\section{Lista de Símbolos}

\begin{tabular}{|c|c|c|}
\hline$A$ & Área & {$\left[\mathrm{m}^{2}\right]$} \\
\hline$b$ & Folga média do canal & [m] \\
\hline Bo & Número de ebulição & \\
\hline$c$ & Razão entre as capacidades caloríficas dos flui & idos \\
\hline$c_{p}$ & Calor especifico a pressão constante & {$[\mathrm{kJ} / \mathrm{kg} \mathrm{K}]$} \\
\hline$C^{*}$ & Capacidade calorífica & {$[\mathrm{kJ} / \mathrm{K}]$} \\
\hline Co & Número convectivo & \\
\hline$D$ & Diâmetro & {$[\mathrm{m}]$} \\
\hline E & Energia & {$[\mathrm{kW}]$} \\
\hline$F$ & Profundidadedo trocador & [m] \\
\hline$f$ & Fator de atrito & \\
\hline $\mathrm{Fr}$ & Número de Froude & \\
\hline$g$ & Aceleração da gravidade & {$\left[\mathrm{m} / \mathrm{s}^{2}\right]$} \\
\hline$G$ & Fluxo mássico & {$\left[\mathrm{kg} / \mathrm{m}^{2} . \mathrm{s}\right]$} \\
\hline $\begin{array}{l}G e_{1}, G e_{2} \\
G e_{3}, G e_{4}\end{array}$ & Parâmetros da correlação de Han et al.(2003) & \\
\hline$h$ & Entalpia especifica & {$[\mathrm{kJ} / \mathrm{kg}]$} \\
\hline$J a$ & Número de Jakob & \\
\hline$k$ & Condutividade térmica & {$[\mathrm{kW} / \mathrm{m} . \mathrm{K}]$} \\
\hline$L$ & Comprimento da placa & [m] \\
\hline$m$ & Massa & {$[\mathrm{kg}]$} \\
\hline$m$ & Vazão mássica & {$[\mathrm{kg} / \mathrm{s}]$} \\
\hline$N$ & Número & \\
\hline $\mathrm{Nu}$ & Número de Nusselt & \\
\hline$N U T$ & Número de unidades de transferência de calor & \\
\hline$p$ & Altura da corrugação & {$[\mathrm{m}]$} \\
\hline$p_{c o}$ & Passo da corrugação & [m] \\
\hline
\end{tabular}




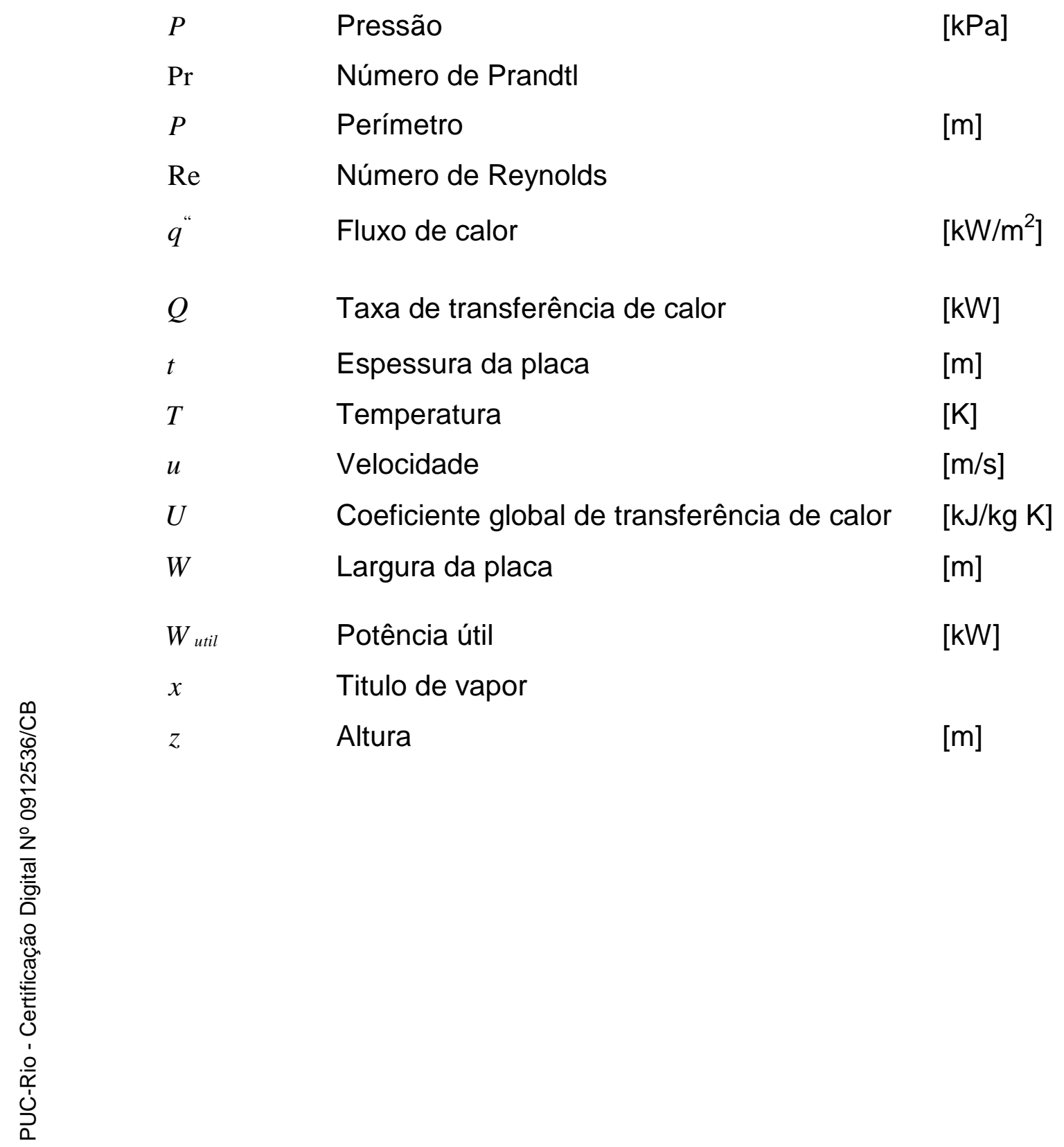




\section{Símbolos gregos}

$\begin{array}{lll}\alpha & \text { Coeficiente de transferência de calor } & {\left[\mathrm{kW} / \mathrm{m}^{2} . \mathrm{K}\right]} \\ \beta & \text { Ângulo de corrugação ou Chevron } & \\ \Delta & \text { Diferença } & \\ \varepsilon & \text { Efetividade } & \\ \phi & \text { Fator de ampliação (dado pelo fabricante) } & \\ \gamma & \text { Fração de vazio } & {[\mathrm{Pa} . \mathrm{s}]} \\ \mu & \text { Viscosidade cinemática } & {\left[\mathrm{kg} / \mathrm{m}^{3}\right]}\end{array}$




\section{Subscritos}

\begin{tabular}{|c|c|c|}
\hline & ave & Médio \\
\hline & $c$ & Critico \\
\hline & $C D$ & Condensador \\
\hline & chan & Canais \\
\hline & cold & Frio \\
\hline & $d i v$ & Divisões \\
\hline & elem & Elemento \\
\hline & $e q$ & Equivalente \\
\hline & $E V$ & Evaporador \\
\hline & $f$ & Atrito \\
\hline 心్రి & $f l u$ & Fluido \\
\hline$\frac{\sqrt{8}}{8}$ & $h$ & Hidráulico, horizontal \\
\hline$\frac{\stackrel{i}{\alpha}}{\bar{\sigma}}$ & hot & Quente \\
\hline 흠 & in & Entrada \\
\hline - & $l$ & Líquido, laminar \\
\hline 衰 & máx & Máximo \\
\hline$\frac{1}{\tilde{n}}$ & $\min$ & Mínimo \\
\hline 仓ે & mom & Momentum \\
\hline & out & Saida \\
\hline & $p$ & Porta \\
\hline & plate & Placa \\
\hline & projetada & Projetada \\
\hline & real & Real \\
\hline & $r e f$ & Refrigerante \\
\hline & sat & Saturação \\
\hline & sta & Estático \\
\hline & $t$ & Turbulento \\
\hline & total & Total \\
\hline
\end{tabular}




$\begin{array}{ll}\text { trans } & \text { Transversal } \\ v & \text { Vapor, vertical } \\ V C & \text { Volume de controle } \\ \text { wall } & \text { Parede }\end{array}$

